\title{
Mecanismo de ação da alcachofra (Cynara Scolymus $L$.) no controle glicêmico: Uma revisão sistemática
}

\author{
Mechanism of action of the artichoke (Cynara Scolymus L.) in glycemic control: A systematic \\ review \\ Mecanismo de acción de La alcachofa (Cynara Scolymus L.) en el control glucémico: Uma revisión
}

sistemática

Recebido: 19/05/2021 | Revisado: 30/05/2021 | Aceito: 31/05/2021 | Publicado: 14/06/2021

\author{
Camila dos Santos Oliveira \\ ORCID: https://orcid.org/0000-0002-0262-885X \\ Universidade José do Rosário Vellano, Brasil \\ E-mail: camila-de-oliveira@outlook.com \\ Catherine Bueno Domingueti \\ ORCID: https://orcid.org/0000-0002-5565-7478 \\ Universidade José do Rosário Vellano, Brasil \\ E-mail: catherine.domingueti@unifenas.br \\ Gérsika Bitencourt Santos \\ ORCID https://orcid.org/0000-0003-0849-2786 \\ Universidade José do Rosário Vellano, Brasil \\ E-mail: gersika.santos@unifenas.br \\ Bruno Cesar Correa Salles \\ ORCID: https://orcid.org/0000-0002-4444-9673 \\ Universidade José do Rosário Vellano, Brasil \\ E-mail: bruno.alfenas@hotmail.com
}

\begin{abstract}
Resumo
O diabetes mellitus gera um grave problema ao sistema de saúde e aos indivíduos, sendo caracterizado por um distúrbio crônico, que afeta o metabolismo de carboidratos, gorduras e proteínas. O presente estudo tem por objetivo realizar uma revisão de literatura demonstrando os mecanismos de ação da alcachofra no controle glicêmico. Foi efetuada uma busca eletrônica noScielo, Pubmed,Science direct e Google Scholar, comparando as diferentes informações encontradas nestas plataformas, listando os mecanismos de ação da alcachofra no controle glicêmico no diabetes mellitus. Foram levantados 63 artigos científicos na língua portuguesa, inglesa e espanhola, dos quais 17,5\% abordaram sobre a alcachofra sua composição, conteúdos fenólicos e efeitos anti-hiperglicêmicos, $13 \%$ descrevem sobre os polifenóis, flavonoides e sua ação anti hiperglicêmica, $16 \%$ relata sobre a regulação da $\alpha$-glicosidase, $6 \%$ aborda sobre o transporte de glicose via GLUT; 6\% sobre a secreção de insulina pelas células beta pancreáticas, $13 \%$ relata a regulação do metabolismo de glicose hepática, $11 \%$ relata a eliminação renal de glicose, 9,5\% sobre o diabetes e suas complicações e $8 \%$ aborda outras plantas e compostos naturais com ação semelhante a alcachofra. De acordo com resultados a possível ação da alcachofra e seus metabólitos especiais está na inibição da alfa-glicosidase, e acrescentar sua importante ação na redução dos radicais livre, redução do glucagon, manutenção hepática do metabolismo glicêmico, proteção das células beta-pancreática, aumento nos níveis de insulina, e captação de glicose nos tecidos, sendo importante fatores na homeostase glicêmica. Por outro lado, a utilização de flavonoides é um dos alvos mais utilizados pela literatura no controle glicêmico a médio e longo prazo. Com a presente revisão é possível concluir que todas as vias mencionadas acima, torna-se algo promissor na busca por tratamento complementar do controle glicêmico de indivíduos portadores do diabetes mellitus.
\end{abstract}

Palavras-chave: Inibição; Cynara Scolymus; Redução da glicemia; Diabetes Mellitus.

\begin{abstract}
Diabetes mellitus generates a serious problem to the health system and to individuals, being characterized by a chronic disorder, which affects the metabolism of carbohydrates, fats and proteins. The present study aims to conduct a literature review demonstrating the mechanisms of action of the artichoke in glycemic control. An electronic search was carried out in Scielo, Pubmed, Science direct and Google Scholar, comparing the different information found on these platforms, listing the mechanisms of action of artichoke in glycemic control in diabetes mellitus. Sixty-three scientific articles were surveyed in Portuguese, English and Spanish, of which $17.5 \%$ addressed artichokes, its composition, phenolic contents and anti-hyperglycemic effects, $13 \%$ describe polyphenols, flavonoids and their antihyperglycemic action, $16 \%$ reports on the regulation of $\alpha$-glycosidase, $6 \%$ addresses glucose transport via GLUT; 6\%
\end{abstract}


on insulin secretion by pancreatic beta cells, $13 \%$ reports the regulation of hepatic glucose metabolism, $11 \%$ reports renal glucose elimination, $9.5 \%$ on diabetes and its complications and $8 \%$ addresses other plants and compounds natural with artichoke-like action. According to the results, the possible action of the artichoke and its special metabolites is in the inhibition of alpha-glucosidase, and add its important action in reducing free radicals, reducing glucagon, liver maintenance of glycemic metabolism, protection of beta-pancreatic cells, increase in insulin levels, and glucose uptake in tissues, being important factors in glycemic homeostasis. On the other hand, the use of flavonoids is one of the most used targets in the literature for glycemic control in the medium and long term. With the present review, it is possible to conclude that all the routes mentioned above become promising in the search for complementary treatment of glycemic control in individuals with diabetes mellitus.

Keywords: Inhibition; Cynara Scolymus; Blood glucose reduction; Diabetes Mellitus.

\section{Resumen}

La diabetes mellitus genera un grave problema para el sistema de salud y para las personas, caracterizándose por un trastorno crónico, que afecta el metabolismo de carbohidratos, grasas y proteínas. El presente estudio tiene como objetivo realizar una revisión de la literatura que demuestre los mecanismos de acción de la alcachofa en el control glucémico. Se realizó una búsqueda electrónica en Scielo, Pubmed, Science direct y Google Scholar, comparando la diferente información encontrada en estas plataformas, enumerando los mecanismos de acción de la alcachofa en el control glucémico en diabetes mellitus. Se recopilaron 63 artículos científicos en portugués, inglés y español, de los cuales el 17,5\% abordó la composición de la alcachofa, su contenido fenólico y efectos antihiperglucémicos, el 13\% describe polifenoles, flavonoides y su acción antihiperglucémica, el 16\% informa sobre la regulación de la $\alpha$ glicosidasa, 6\% aborda el transporte de glucosa a través de GLUT; El 6\% sobre la secreción de insulina por las células beta pancreáticas, el 13\% informa sobre la regulación del metabolismo hepático de la glucosa, el 11\% informa sobre la eliminación renal de la glucosa, el 9,5\% sobre la diabetes y sus complicaciones y el $8 \%$ se ocupa de otras plantas y compuestos naturales con acción similar a la alcachofa. Según los resultados, la posible acción de la alcachofa y sus metabolitos especiales está en la inhibición de la alfa-glucosidasa, y sumar su importante acción en la reducción de radicales libres, reducción de glucagón, mantenimiento hepático del metabolismo glucémico, protección de betacélulas pancreáticas, aumento de los niveles de insulina y captación de glucosa en los tejidos, factores importantes en la homeostasis glucémica. Por otro lado, el uso de flavonoides es una de las dianas más utilizadas en la literatura para el control glucémico a medio y largo plazo. Con la presente revisión, es posible concluir que todas las vías mencionadas anteriormente, se convierte en algo prometedor en la búsqueda de tratamientos complementarios de control glucémico en personas con diabetes mellitus.

Palabras clave: Inhibición; Cynara Scolymus; Reducción de glucosa en sangre; Diabetes Mellitus.

\section{Introdução}

As doenças crônicas não transmissíveis (DCNT) causam atualmente aproximadamente 16 milhões de mortes prematuras (World Health Organization, 2014). A Federação Internacional de Diabetes (IDF) estimou que em 2017 cerca de $8,8 \%$ da população mundial entre 20 a 79 anos de idade vivia com diabetes, neste estudo o Brasil se posicionou em quarto lugar com cerca de 12,5 milhões de pessoas com esta doença (Lyra et al., 2020). Segundo o relatório anual da Organização Mundial de Saúde (OMS) publicado em 2014, as cardiopatias e pneumopatias, os acidentes cerebrovasculares, o câncer e o diabetes são os grandes responsáveis por elevados índices de mortes prematuras (World Health Organization, 2014). No Brasil, estudos apontam para três grandes grupos responsáveis pela "epidemia de DCNT", o diabetes, a doença cardiovascular e o acidente vascular encefálico (Malta \& Silva Jr, 2013).

O diabetes Mellitus é caracterizado por um distúrbio crônico, que afeta o metabolismo de carboidratos, de gorduras e proteínas. O diabetes Mellitus é considerado um problema de saúde pública, pois está associado a diversas complicações sérias para o paciente como alterações microvasculares, na qual se pode citar a retinopatia, neuropatia e nefropatia, e complicações macrovasculares que são observadas, incluindo doença cardíaca coronária e acidente vascular cerebral.

A vida vegetal tem sido alvo de frequentes e volumosos estudos recentemente devido à grande possibilidade da descoberta de novos compostos bioativos que poderiam auxiliar na cura ou tratamento de enfermidades (Viegas Jr et al., 2006). Segundo dado da OMS (Organização Mundial de Saúde) cerca de $80 \%$ da população mundial já fez uso de algum tipo de erva na busca do alívio de alguma enfermidade. 
Muitos estudos têm demonstrado que plantas medicinais vem sendo eficazes no tratamento das complicações crônicas do diabetes Mellitus, assim como no controle glicêmico. No entanto, os mecanismos biológicos responsáveis por tais efeitos ainda não foram elucidados. O teor de metabólitos secundários, as vias de sinalização intracelular, a absorção intestinal, as classes químicas dos metabólitos secundários, poderão influenciar nos mecanismos mencionados pela literatura.

Os mecanismos de ação hipoglicemiante de produtos vegetais têm sido alvo de estudo por diversos grupos de pesquisa. Cada classe química de metabólitos especiais possui uma característica peculiar que irá influenciar em seu mecanismo de ação. Portanto, conhecer os possíveis mecanismos de ação de produtos de origem vegetal no controle glicêmico, torna-se alvo de muito interesse científico.

\section{Metodologia}

O trabalho realizado não envolveu o uso de dados de seres humanos, não necessitando de qualquer tipo de avaliação clínico-laboratorial e, assim, não foi necessário a aprovação pelo Comitê de Ética. O estudo trata-se de uma pesquisa bibliográfica sistemática, constituída de livros e artigos científicos (Pereira et al., 2018). Foi efetuado uma revisão de literatura sobre o tema em revistas acadêmicas científicas e artigos, através de bancos de dados, tais como Scielo, Pubmed, Science direct e Google Scholar, de modo que se comparou as diferentes informações encontradas nestas plataformas, listando as principais condições que aborda os mecanismos de ação da alcachofra no controle glicêmico do diabetes Mellitus. Foram pesquisados artigos utilizando as seguintes descrições: diabetes Mellitus, alcachofra (Cynara Scolymus L.), efeitos antihiperglicêmicos dos flavonóides, regulação da $\alpha$-Glicosidase, transporte de Glicose, secreção de Insulina, metabolismo glicose hepática, aumento da eliminação renal. Os critérios de inclusão foram: artigos publicados entre 2000 e 2021, originais, em língua portuguesa e inglesa, que obedeciam às palavras chaves descritas, tal como apresentado no Quadro 1.

Quadro 1. Métodos de busca bibliográficas.

\begin{tabular}{|c|c|c|c|}
\hline Banco de dados & Descritores & Critérios de aceitação & $\begin{array}{l}\text { Total de } \\
\text { artigos }\end{array}$ \\
\hline $\begin{array}{l}\text { Diabetes Spectrum } \\
\text { Google Scholar } \\
\text { Elsevier } \\
\text { Europe PMC } \\
\text { Frontiers in Endocrinology. } \\
\text { Johns Hopkins University. } \\
\text { MDPI } \\
\text { Oxford Academic } \\
\text { Pubmed } \\
\text { Researchgate } \\
\text { Scielo } \\
\text { Science Direct } \\
\text { Scopus } \\
\text { Sage Journals } \\
\text { Springer Nature } \\
\text { Unifal } \\
\text { Wiley online Library }\end{array}$ & $\begin{array}{l}\text { Diabetes Mellitus; } \\
\text { Alcachofra (Cynara Scolymus L.); } \\
\text { Efeitos anti-hiperglicêmicos dos } \\
\text { flavonóides; } \\
\text { Regulação da } \alpha \text {-Glicosidase; } \\
\text { Transporte de Glicose; } \\
\text { Secreção de Insulina; } \\
\text { Metabolismo Glicose Hepática; } \\
\text { Aumento da eliminação Renal. }\end{array}$ & $\begin{array}{l}\text { Artigos publicados entre } 2000 \text { e } \\
2021 . \\
\text { Originais; } \\
\text { Na língua Portuguesa, Inglesa e } \\
\text { Espanhola. }\end{array}$ & 63 \\
\hline
\end{tabular}

Fonte: Autores.

\section{Resultados e Discussão}

No presente estudo Foram levantados 63 artigos científicos na língua portuguesa, inglesa e espanhola, dos quais $17,5 \%$ abordaram sobre a alcachofra (Cynara Scolymus L.) sua composição, conteúdos fenólicos e efeitos anti-hiperglicêmicos, 13 \% 
descrevem sobre os polifenóis, flavonoides e sua ação anti hiperglicêmica, $16 \%$ relata sobre a regulação da $\alpha$-glicosidase, $6 \%$ aborda sobre o transporte de glicose via GLUT; 6\% sobre a secreção de insulina pelas células beta pancreáticas, 13\% relata a regulação do metabolismo de glicose hepática, $11 \%$ relata sobre a eliminação renal de glicose, 9,5\% sobre o diabetes e suas complicações e $8 \%$ aborda outras plantas e compostos naturais com ação semelhante a alcachofra.

De acordo com Mishra et al. (2017) o diabetes afeta milhares de pessoas, sendo ocasionado pela falta ou deficiência do hormônio insulina, responsável em regular os níveis de glicose no sangue (Mishra et al., 2017). Sendo 80 a $90 \%$ dos casos relacionados à diabetes Mellitus (tipo 2) que comprometem o metabolismo de carboidratos, gorduras e proteínas, esses fatores são interligados à crescente prevalência de obesidade e sedentarismo (Lyra et al., 2020).

O diabetes Mellitus frequentemente gera cegueira, amputação, insuficiência renal, dano neural, ataques cardíacos e acidentes vasculares encefálicos (Ferrier, 2018). Durante 2006 estudos foram realizados estimando que até 2030, seriam 439 milhões de pessoas atingidas com essa endocrinopatia (Huizinga; Rothman, 2006). Entretanto, estudos atuais revelam que em 2019, esses resultados foram ultrapassados com 463 milhões de pessoas (Idf, 2019).

Desse modo o presente estudo abordou as ações da alcachofra (Cynara Scolymus L.) na qual apresentam importante ação antioxidantes, redução glicêmica, anticarcinogênica, antiinflamatória, hepatoprotetora, cardioprotetoras, diuréticas, antimicrobianas, redução do colesterol, regulação na liberação da bile, inibição da biossíntese de colesterol e oxidação do LDL (Lattanzio et al., 2009). Os extratos das folhas de Cynara Scolymus foram relacionados a tratamento de distúrbios metabólicos e apontam com uma boa fonte de polifenóis (Ben Salem et al., 2017).

Os polifenóis são encontrados nas plantas e apresentam atividades antioxidantes, anti-hipertensiva e inibidores da alfaglicosidase. Apresentam compostos aromáticos que carregam um ou mais grupos hidroxila, que são divididos em ácidos fenólicos e flavonóides (Ghani, 2020b).

Uma das alternativas que resultam na melhora dos índices glicêmicos são as plantas que atuam na inibição de enzimas digestivas tal como a alfa-glicosidase evitando a conversão em açúcares simples que serão absorvidos pelo intestino (Ben Salem et al., 2017). Assim como também a regulação via GLUT, responsável pelo transporte de glicose para dentro das células.

Outra ação importante é a regulação na secreção de insulina, visto que o descontrole entre a ação e a secreção da insulina resulta em aumento da glicose. Em longos períodos essa hiperglicemia e hiperlipidemia podem resultar em falência ou morte das células beta-pancreática (Christensen \& Gannon, 2019).

Observa-se também a importância da regulação do metabolismo de glicose hepática visto que em pacientes com diabetes Mellitus (tipo 2), a glicose hepática é produzida de maneira elevada podendo levar a picos de hiperglicemia (Klover \& Mooney, 2004).

Além disso, é importante destacar a ação dos flavonóides na eliminação de glicose renal, uma vez que a inibição do cotransportador de sódio-glicose (SGLT-2) presente nos rins favorece a eliminação de glicose renal e auxilia na redução de glicose no sangue (Pires, 2017).

\subsection{Alcachofra (Cynara scolymus L.)}

As plantas são tradicionalmente usadas há bastante tempo na busca de cura, tratamento ou alívio de alguma enfermidade sendo mais utilizadas para acnes, aftas, anemia, asma, colesterol, diabetes, enxaqueca, febre, gastrite (Braga, 2011).

A Cynara Scolymus é uma planta antiga de origem da Bacia do Mediterrâneo, possui propriedades antioxidantes, antihiperglicêmica, anti-carcinogênicas, anti-inflamatórias, hepatoprotetoras, cardioprotetoras, diuréticas, antimicrobianas e redutoras de colesterol (Zuorro et al., 2016). 
Segundo Grandi (2014), ela é uma planta herbácea perene, pertencente à família Asteraceae que atinge $1 \mathrm{~m}$ de altura, sensível a geada, apresentam caule esbranquiçado, as folhas são grandes e verde-claras, lanceoladas, carnosas, pubescentes e aparência recortada. Seu pecíolo e a nervura principal são muito salientes, quando se tornam adulta, eleva-se um pedúnculo cilíndrico, com poucas e pequenas folhas. Nas extremidades, surgem capítulos recobertos de brácteas coriáceas de cor roxa, tornando-se depois pálidas ou quase brancas. Suas flores são azuis de corola estreitamente tubulosas, longas, com 5 lobos lineares na extremidade. Seus frutos apresentam uma coroa de cerdas pilosas (Grandi, 2014). De acordo com a Figura 1, demonstra esquema representativo da planta alcachofra e suas partes (Saucier, 2013).

Figura 1. Alcachofra com suas partes.

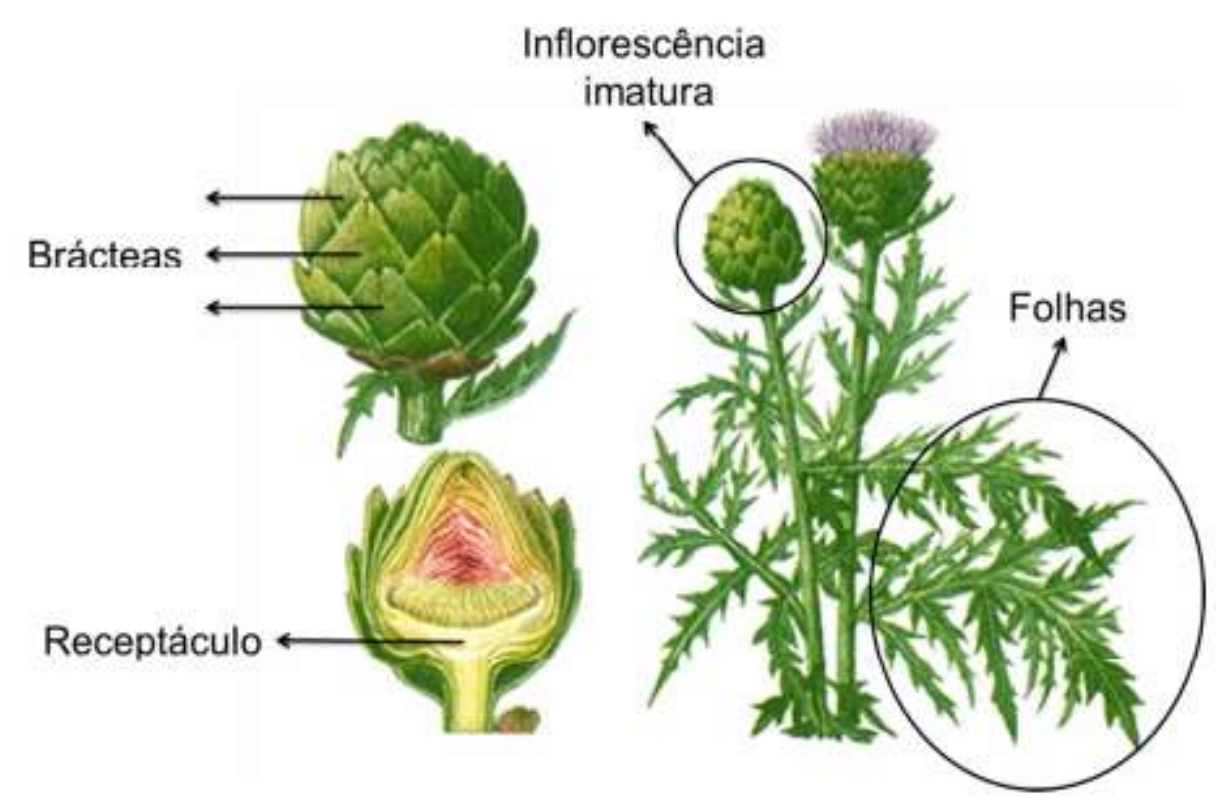

Fonte: Saucier, (2013).

Seu nome botânico que é derivado do Latim cinis, cineris, por causa da tradição de usar cinzas como fertilizante, e do grego skolymos, que significa cardo devido aos espinhos localizados nas brácteas que circundam a inflorescência, como parte da parte comestível da planta (Frutos et al., 2018). Possui altos níveis de minerais como potássio, cálcio e sódio, além de magnésio, fósforo, ferro, cobre e manganês. Apresenta ainda vitamina C, folato, fibras, polifenóis, flavonas, inulina e hidroxicinamatosácidos cafeoilquínicos e derivados (Frutos et al., 2018).

Segundo Fantini et al. (2011), o extrato de cabeça da flor de Cynara scolymus apresentou redução da glicemia pósprandial em ratos Wistar e ratos Zucker obesos, e como não apresentavam qualquer conteúdo de fibra nesta cabeça de flor do extrato, foi excluído o envolvimento das fibras dietéticas na redução da glicemia (Fantini et al., 2011).

De acordo com Salem et al. (2015), os efeitos da alcachofra no controle da hiperglicemia podem estar relacionados à presença de compostos, como terpenos, polifenóis, flavonóides, taninos e compostos de glicosídeos cardíacos (Salem et al., 2015).

Os taninos hidrolisáveis estão presentes nas pétalas da Rosa galica, que são frequentemente usados na China no tratamento do diabetes Mellitus. A presença de taninos na alcachofra pode estar relacionada com inibição da alfa-glicosidase, pois recentemente surgiu na literatura sobre a presença de taninos na inibição da alfa-glicosidase (Muccilli et al., 2017).

De acordo com Rezazadeh \& Ebrahimi-Mameghani (2020), os extratos da alcachofra apresentam compostos bioativos importantes como polifenóis, triterpenos e lactonas. Os principais compostos polifenólicos são ácidos cafeoilquínicos (mono- e 
diésteres) e flavonóides. A Cynara scolymus possui também ácidos hidroxicinâmicos (ácido clorogênico, ácidos cafeoilquínicos, ácido cafeico, ácido ferúlico), flavonóides (luteolina e escoliosida e cynaroside), cynarin (ácido 1,5-di-cafeoilquínico), são os principais ingredientes do extrato da alcachofra (Mahboubi, 2018).

No estudo realizado por Dragan et al. (2014) apontam os derivados do ácido fenólico (Cafeico, ácidos ferulicclorogênicos, cinarina, p-cumárico, ácido vanílico gálico, ácidos p hidroxibenzóico) como compostos naturais com efeitos antidiabéticos presentes na Cynara scolymus $L$.

Os ácidos cafeoilquínicos são identificados no extrato da alcachofra, encontram em maior quantidade ácido clorogênicos (5-O-cafeoilquínico), depois são os ácidos 1,5-O dicafeoilquínico e de 3,4-O- ácido dicafeoilquínico. A Cynarina (ácidos 1,3-O dicafeoilquínico) é o mais conhecido derivado cafeoilquínico encontrado na alcachofra, no entanto não é o mais abundante (Lattanzio et al., 2009).

De acordo com Salem et al. (2015), apresenta ainda outros compostos fenólicos como as flavonas 5,7-dihidroxi-2-(4hidroxifenil) -4H1-benzopiran-4-ona(apigenina) e 2-(3,4- dihidroxifenil) - 5,7-dihidroxi-4-cromenona (luteolina), bem como o antocianidinas, tais como 2-(3,4-dihidroxifenil) cromenilio-3,5,7-triol (cianidina), 2- (4-hidroxi-3 metoxifenil) cromenílio-3,5,7triol (peonidina) e 2- (3,4,5-trihidroxifenil) cromenilio-3,5,7-triol (delfinidina) foram isoladas apenas nas cabeças de alcachofra.

Os extratos da alcachofra contêm bioativos e flavonóides, como ácidos cafeoilquínicos e glicosídeos de luteolina. O extrato de cabeça da flor da Cynara scolymus L. obteve redução da glicemia pós-prandial em ratos Wistar e ratos Zucker obesos geneticamente selecionados (Fantini et al., 2011).

Os flavonóides são compostos bioativos importantes presentes nas plantas e incluem flavonóis, flavonas, isoflavonas, flavano, catequinas (Flavan-3-ols), antocianinas e chalconas (Rastija et al., 2012).

A inibição de enzimas digestivas como alfa-glicosidase e alfa-amilase reduz a velocidade de digestão e absorção dos carboidratos. Assim os níveis de glicose no sangue pós prandial ficam controlados (Taslimi \& Gulçin, 2017). Na pesquisa de Taslimi \& Gulçin (2017), todos os compostos fenólicos naturais usados tiveram efeitos inibitórios eficazes da alfa- glicosidase do que o da acarbose.

Segundo o mesmo autor, os compostos fenólicos naturais, apresentam relação com a propriedades inibitórias in vitro das enzimas metabólicas como alfa-amilase e alfa-glicosidase relacionadas com o diabetes Mellitus (Taslimi \& Gulçin, 2017).

\subsection{Regulação da $\alpha$-glicosidase}

As glicosidases são enzimas que fazem parte das classes de enzimas hidrolases, que clivam ligações glicosídicas para liberação de glicose. Estudos demonstram que a $\alpha$-glicosidase está presente no intestino delgado têm papéis importantes no metabolismo dos carboidratos e sua inibição é um método terapêutico atraente para o tratamento de diabetes e obesidade (Santos et al., 2018). As $\alpha$-glicosidases quebram ligações $\alpha$-glicopiranosídica (alfa- 1,4) em carboidratos complexos para liberação de glicose. $\mathrm{O}$ extrato da alcachofra contém apigenina-7-glicosideo e apigenina sendo relacionado com a inibição de alfa-amilase e alfa-glicosidase. Apontam que a inibição da $\alpha$-glicosidase pode reduzir à absorção de carboidratos e diminuir a hiperglicemia (Ben Salem et al., 2017).

No estudo realizado por Sheliya et al. (2015), a Euphorbia hirta L. é utilizada no tratamento da diabetes, seu objetivo foi isolar e caracterizar os constituintes presentes na planta e sua capacidade inibição in vitro da $\alpha$-glucosidase.

Esse potencial inibidor da $\alpha$-glicosidase de compostos isolados foi avaliado e comparado com o medicamento padrão acarbose (Sheliya et al., 2015). Sua inibição foi determinada pela análise do gráfico de Lineweaver-Burk. Assim para Sheliya et 
al. (2015), flavonóides prenilados presentes em E. hirta apresentam capacidade de inibição da $\alpha$-glicosidase com possibilidade do uso para o manejo do diabetes Mellitus.

Para Fernandes (2020), o perfil estrutural e outras características dos polifenóis, e peso molecular número e posição de substituição e glicosilação de flavonóides, parecem estar relacionados com o efeito inibitório da enzima digestiva.

As enzimas geralmente são isoladas a fim de realizar teste in vitro, podem ser reguladas, ou seja podem ser ativadas ou inibidas, para corresponder às necessidades das células. Podem agir de diferentes formas dependendo da concentração de substrato, temperatura e pH (Ferrier, 2018).

As glicosidases, incluindo $\alpha$-glicosidases ( $\alpha$-D'Glucosídeo glucohidrolase; EC 3.2.1.20) são um grupo de enzimas que apresentam papéis fundamentais no metabolismo de carboidratos e no processamento de glicoproteínas (Ernst et al., 2006). A $\alpha$ glicosidases hidrolisam especificamente o ligação $\alpha$-glicopiranosídeo $(\alpha-1,4)$ em carboidratos complexos para liberar glicose (Ghani, 2020a).

A alfa-glicosidase apresentam funções nos mamíferos como glicogênio degradação, processamento de oligossacarídeos ligados a N para dobramento e maturação de glicoproteínas e digestão intestinal de carboidratos dietéticos (Ghani, 2020a, p.6).

Os inibidores são substâncias que vão interferir na ação das enzimas e diminuir a velocidade de reação. Informações importantes podem ser encontradas observando reações enzimáticas e analisando sua reação na presença de um inibidor (Campbell \& Farrell, 2016).

Os inibidores da $\alpha$-glicosidase com características anti-diabéticas possuem semelhanças estruturais ao açúcar, são usados como uma ferramenta que modificam aspectos funcionais. Sua estrutura contém anel com grupo amina e alguns substituintes hidroxila dispostos de maneiras tridimensionais são semelhantes à estrutura básica de carboidratos (Mishra et al., 2017, p.153).

Os inibidores reversíveis da alfa-glicosidase se ligam de modo reversível na alfa-glicosidase presente no intestino delgado evitando a hidrólise de oligossacarídeo e reduz glicose no sangue (Mishra et al., 2017, p.154).

Os inibidores irreversíveis da glicosidase, apresentam a gasternospermina que são inibidores potentes de sacarose que incluem voglibose, que é administrada para tratar diabetes mellitus na condição pós-prandial. Eles mantêm glicemia mais baixa, facilitando a absorção retardada de glicose (Mishra et al., 2017, p.154). De acordo com a Figura 2, representa atividade de $\alpha$ glicosidase intestinal e inibição da $\alpha$-glicosidase pelos produtos naturais. 
Figura 2. Representa a atividade de $\alpha$-glicosidase intestinal e inibição da $\alpha$-glicosidase pelos produtos naturais.

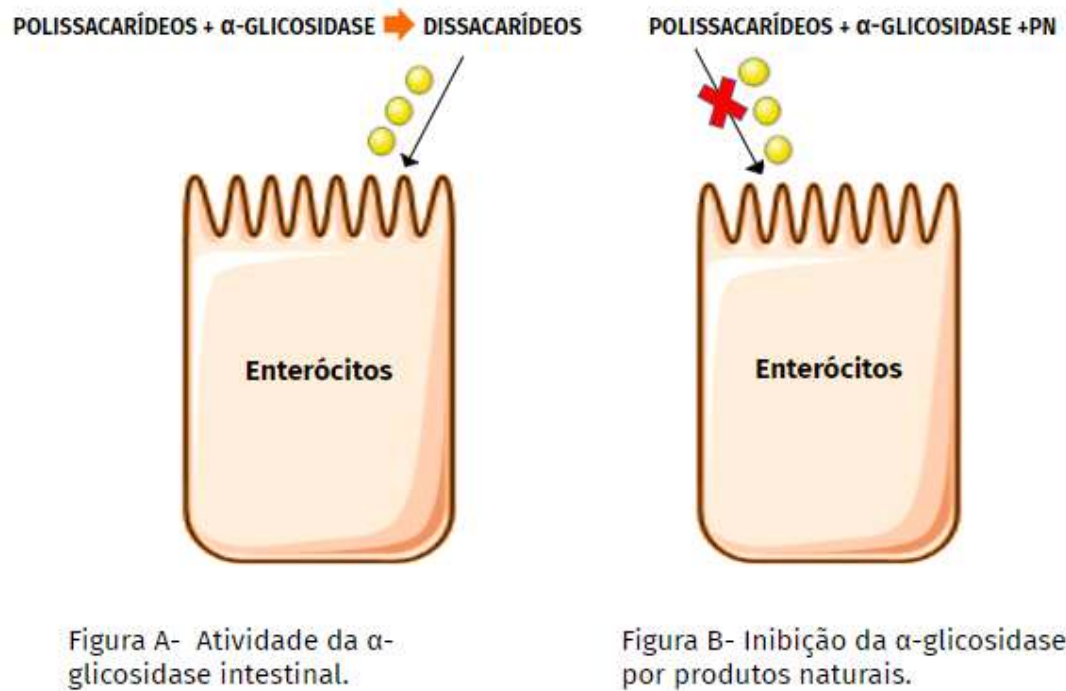
glicosidase intestinal. por produtos naturais.

Fonte: Adaptada de Rosak \& Mertes (2012).

A enzima está presente no intestino delgado, é importante na digestão e absorção de carboidratos. Realiza a quebra de polissacarídeos e oligossacarídeos em unidades menores para que sejam absorvidas, aumentando a concentração de glicose no corpo (Abbas et al., 2017).

Sua inibição retarda a digestão e absorção de carboidratos, assim a glicose no sangue pós-prandial é mantida em níveis mais reduzidos, levando a uma diminuição da necessidade de insulina (Abbas et al., 2017).

Alguns fenólicos foram isolados para atividade de $\alpha$-glicosidase, testando sua atividade. Entre estes compostos, com unidade de glicosil ligada foram encontrados e têm atividade inibidora de $\alpha$-glicosidase; que diminuem os níveis de glicose pósprandial no sangue (Mishra et al., 2017). Estudos apontam a eficácia dos flavonóides como inibidores da glicosidase, que são abundantes em plantas naturais e possuem propriedades farmacológicas.

Diversas concentrações do inibidor são usadas para determinar o IC50 (concentração na qual 50\% de inibição é observada). Os bioensaio resultaram na identificação de algumas classes de produtos naturais como flavonóides, terpenos, alcalóides, quinonas e polifenóis com atividade antidiabética aprimorada (Abbas et al., 2017, p.265).

\subsection{Transporte de glicose}

Em condições normais após o consumo de (carboidratos, açúcar, gorduras) as moléculas são digeridas e liberadas em partículas menores na corrente sanguínea. A glicose juntamente com cálcio ativa as células beta-pancreáticas para liberação de insulina que é o principal hormônio responsável pela homeostase glicêmica (Al-Ishaq et al., 2019).

A insulina liberada e glicose presentes na corrente sanguínea serão metabolizadas nas células. As células apresentam receptores que são ativadas insulina, desencadeando uma cascata de sinalização intracelular com ativação do Substrato receptor de insulina (IRS) culminando com a translocação do transportador de glicose (GLUT 4) para a membrana plasmática de acordo com Figura 3 (Carvalheira et al., 2002).

As proteínas GLUT são codificadas pelos genes SLC2, a proteína de transporte GLUT4 fica responsável por transportar glicose para dentro das células, no entanto é necessário de estímulos mediados pela insulina, sendo assim resistência à insulina e 
defeito no GLUT4 é a principal causa do diabetes tipo 2 (Mueckler \& Thorens, 2013). O aumento na resistência à insulina diminui a translocação de GLUT, diminui captação e absorção de glicose (Al-Ishaq et al., 2019).

Figura 3. Demonstração captação de glicose por GLUT 4.

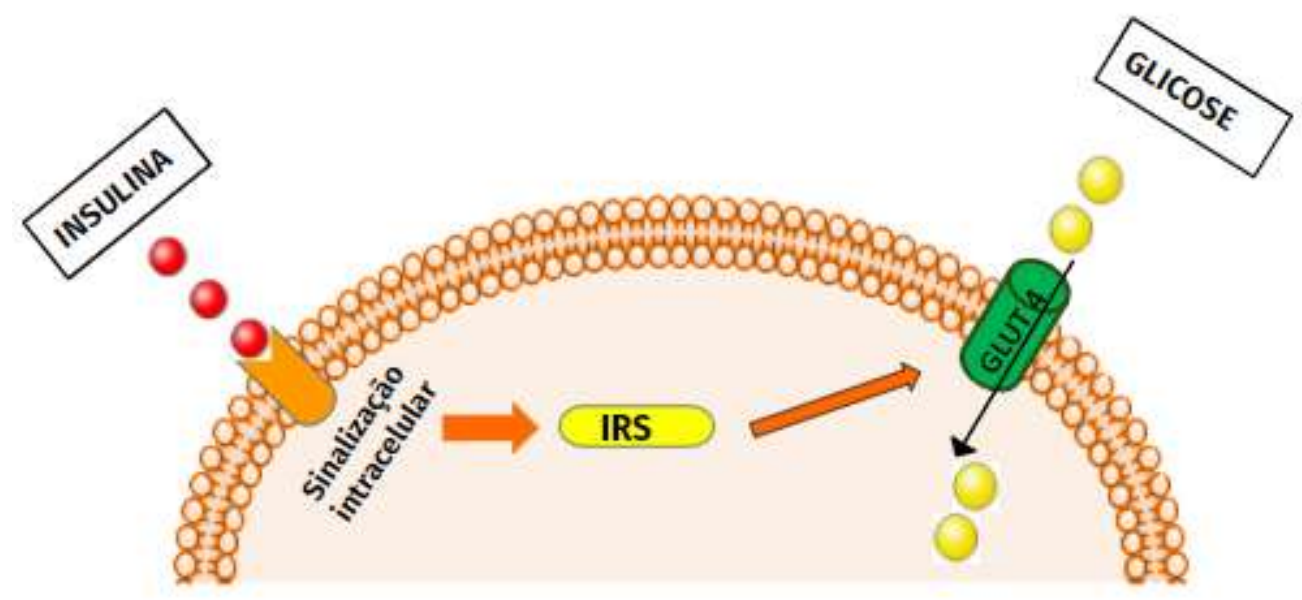

Fonte: Adaptada de Salles et al. (2019).

Segundo Mejri et al. (2020), a apigenina presente na alcachofra são benéficas na redução glicêmica, protegendo as células beta-pancreáticas de danos oxidativos, aumenta a secreção de insulina e possibilita maior captação de glicose pelos transportadores (GLUT).

No diabetes (tipo 2) acontece uma resistência à insulina devido a produção anormal de insulina e os altos índices de hiperglicemia geram alterações no tamanho e função das células betas pancreáticas sendo incapaz de atuar com altas demandas de insulina (Al-Ishaq et al., 2019).

Segundo Mahboubi (2018), a Cynara Scolymus inibe as enzimas digestivas, como a lipase do pâncreas, $\alpha$-amilase, $\alpha$ glicosidase, aumenta a secreção biliar, inibe a inflamação e estresse do retículo endoplasmático, melhora a função hepática, a microbiota intestinal, aumenta a lipólise, metabolismo lipídico e reduz a glicose no sangue.

De acordo com Salles et al., (2020) os flavonóides apresentam capacidade de atuar reduzindo marcadores bioquímicos. No seu estudo os extratos de Passiflora edulis foram capazes de reduzir glicose, creatinina, colesterol total e colesterol não HDL no soro e no sangue HbA1. Os extratos apresentavam quercetina-3-O- $\beta$-glicopiranosil-7-O- $\alpha$-ramnopiranosídeo e quercetina-8O- $\beta$-glicopiranosil-6-O- $\alpha$-ramnopiranosídeo, 6,8-Di-C- $\beta$-glicopiranosil apigenina (vicenina-2), apigenina-6-C-arabinosídeo-8-Cglicosídeo, isoorientina, isovitexina, ácido 4-O- $\beta$-glicopiranosil cafeico, ácido fenólico e derivado de ácido fenólico (Salles et al., 2020).

Segundo o mesmo autor, os polifenóis apresentam ações importantes na redução da absorção de açúcar, aumentam a captação de glicose nos músculos esqueléticos e tecido adiposo e reduz os níveis de glicose no sangue (Salles et al., 2020).

\subsection{Secreção de insulina}

O descontrole entre a ação e a secreção da insulina resulta em aumento da glicose no sangue. A secreção de insulina acontece pelas células beta-pancreática, assim uma disfunção nas células beta, a secreção de insulina é reduzida, não sendo capaz de manter os níveis de glicose (Galicia-Garcia et al., 2020). A preservação das células beta, sinalização da insulina nas células beta e a sinalização da insulina nos tecidos receptores de glicose manterão a homeostase da glicose (Cerf, 2013). 
O mau funcionamento das células betas em pacientes portadores de diabetes Mellitus (tipo 2) pode estar relacionado com diversos fatores entre eles a hiperglicemia e dislipidemia que favorecem a resistência à insulina, inflamação crônica, estresse do retículo endoplasmático, estresse metabólico e pode levar a falência das células betas. Esse excesso de ácidos graxos livres e hiperglicemia levam a perda de função das células beta-pancreáticas, induz ao estresse do retículo endoplasmático e liberação proteínas apoptóticas (Christensen \& Gannon, 2019).

A insulina quando pronta é armazenada em grânulos até que seja acionada sua liberação. Essa liberação acontece devido a altas concentrações de glicose, quando os níveis de glicose aumentam, as células betas absorvem glicose através de transportadores GLUT2, e outros fatores também ativam a liberação de insulina como aminoácidos, ácidos graxos e hormônios (Galicia-Garcia et al., 2020).

Uma questão importante é sobre a capacidade da alcachofra em promover ação protetora das células $\beta$ do pâncreas em ratos diabéticos aloxanos, em comparação com acarbose (Ben Salem et al., 2017).

Na Figura 4, demonstra o esquema da progressão T2D. A resistência insulina em longo tempo e inflamação deixam as células beta estressadas e descompensadas, levando a tolerância à glicose diminuída. A hiperglicemia e hiperlipidemia em curso levam as células beta a uma glicolipotoxicidade e falência das células $\beta$. A massa funcional das células beta diminui devido à desdiferenciação e / ou morte de células $\beta$, levando ao diabetes tipo 2 (Christensen \& Gannon, 2019).

Figura 4. Esquema da progressão T2D.

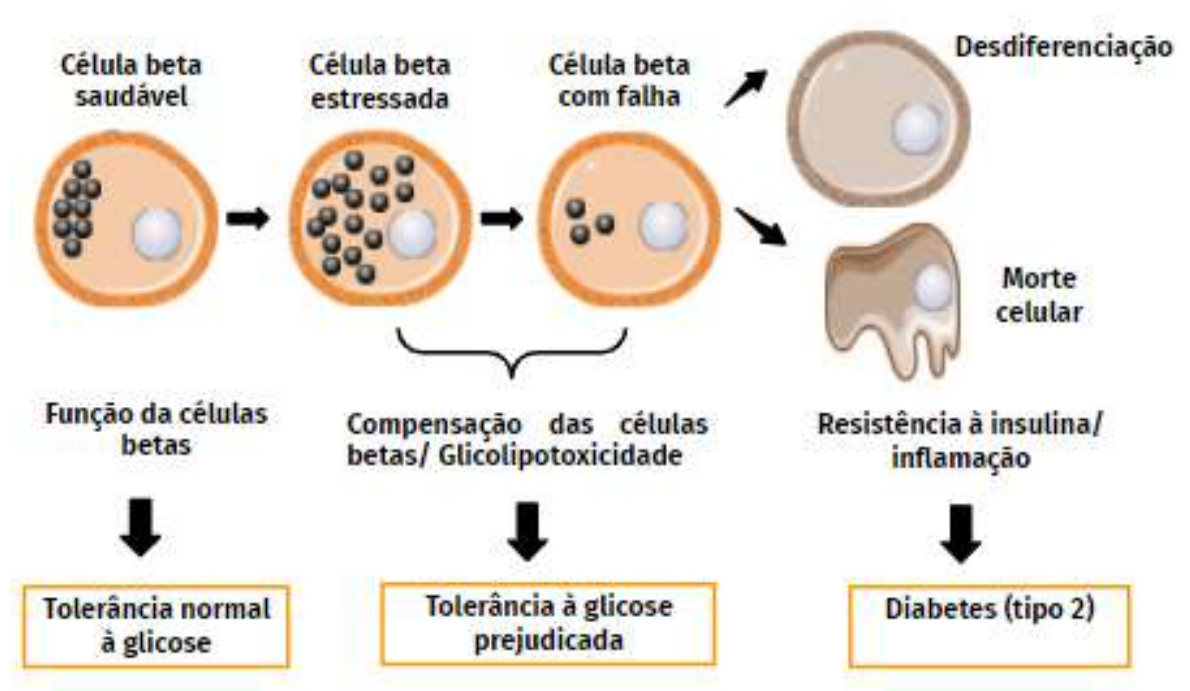

Fonte: Adaptada de Christensen \& Gannon (2019).

No entanto, o uso de plantas medicinais já vem sendo usado há bastante tempo, a alcachofra é uma planta importante estudada por diversos trabalhos, sendo abordada devido à capacidade de atuarem no diabetes mellitus. Uma das ações da alcachofra é na proteção das células $\beta$ pancreáticas foi demonstrado que os ácidos cafeoilquínicos presentes na alcachofra, como o ácido feoilquínico 5-O-caf; Ácido 3,4-dicafeoilquínico; Ácido 3,5-dicafeoilquínico; Ácido 4,5-dicafeoilquínico; e seus derivados de metila e etila são os principais responsáveis pela proteção das células $\beta$ pancreáticas, estimulação da secreção de insulina e inibição de $\alpha$-glicosidase (Mejri et al., 2020). 
No estudo de Hosseini et al. (2015) os extratos hidroalcoólicos da alcachofra foram responsáveis por reduzir glucagon, glicose, ALT, AST, ALP e aumentar os níveis de insulina. De acordo com o autor, a alcachofra é responsável pela proteção das células beta-pancreática e reduz radical livre devido à ação de compostos presentes como ácido clorogênico, cinarina e luteolina.

Esses compostos importantes também são responsáveis por efeitos semelhantes à insulina aumentando a captação de glicose nos tecidos, são capazes de inibir enzimas digestivas responsáveis absorção de carboidratos, reparam danos celulares e aumenta células beta-pancreáticas melhorando a secreção de insulina (Hosseini et al., 2015).

\subsection{Metabolismo glicose hepática}

O fígado é o maior reservatório de glicose em nosso corpo, armazena glicose em forma de glicogênio, essa ação de várias glicose transformando em glicogênio é chamada de glicogênese (Moore et al., 2012). No entanto, essa ação é influenciada pelo hormônio insulina, para ocorrer estocagem de glicose e ser usada quando o corpo necessite de glicose.

O glucagon é outro hormônio produzido no fígado, no entanto pelas ilhotas alfa pancreáticas, esse hormônio auxilia quando a pessoa apresenta picos hipoglicêmicos. Estimula à quebra de glicogênio hepático acontecendo a glicólise, essa ação é chamada de glicogenólise e a glicose liberada será levada para a corrente sanguínea suprindo nos quadros de hipoglicemia (Aronoff et al., 2004).

Quando a reserva de glicogênio está acabando o fígado estoca mais glicose essa reação é influenciada pelo glucagon que leva a ação de gliconeogênese, nova formação de glicose (Aronoff et al., 2004). Assim fígado é extremamente importante na regulação glicêmica visto atuando tanto em quadro de hiperglicemia fazendo a estocagem de glicose e em estado de hipoglicemia fazendo liberação de glicose (Klover \& Mooney, 2004).

No entanto, a resistência à insulina em quadros de diabetes (tipo 2) gera prejuízo nas vias de homeostase glicêmicas hepática. A glicogenólise e a gliconeogênese, atuam de forma ineficiente em resposta à insulina podendo levar à liberação inadequada de glicose pelo fígado e resultados na hiperglicemia, que pode ocorrer após as refeições e durante o jejum (Klover \& Mooney, 2004).

De acordo com estudo de Mejri et al. (2020), as alcachofras foram benéficas no tratamento de camundongos diabéticos, são ricas em ácidos cafeoilquínicos e flavonas, são capazes de reduzir a glicose no sangue, reduz marcadores hepáticos como alanina aminotransferase, aspartato aminotransferase, fosfatase alcalina, creatinina e atuam como antioxidante hepáticos e renal. Além disso, foram capazes de reduzir o colesterol total, triglicerídeos, colesterol LDL que são fatores de risco para aterosclerose e doenças cardiometabólicas (Mejri et al., 2020).

De acordo com El Sayed et al. (2018), o extrato da alcachofra é capaz de exercer proteção do fígado e atividade de redução do colesterol. No estudo histopatológico realizado em fígado de ratos, o extrato da alcachofra no nível de dose 500 $\mathrm{mg} / \mathrm{kg}$ ao dia foi capaz de reduzir lesões hepáticas (El Sayed et al., 2018).

No estudo Abdel-Salam et al., (2013) ratos foram induzidos a lesão hepática com tetracloreto de carbono (CC14) e após serem tratados com extrato de Cynara a 60 e $120 \mathrm{mg} / \mathrm{kg}$ apresentavam uma proteção significativa contra danos hepatotóxicas do CC14, reduzindo alanina aminotransferase sérica (ALT), aspartato aminotransferase (AST) e fosfatase alcalina. Em estudo histopatológico notaram que a lesão hepática induzida foi reduzida após tratarem com extrato de alcachofra. Segundo o autor, a Cynara pode ser útil no tratamento de doenças hepáticas crônicas (Abdel-Salam et al., 2013).

De acordo com Hanhineva et al. (2010) a resistência à insulina é uma interferência no metabolismo e produção de glicose hepática. O fígado fica incapaz de responder à insulina gerando alterações na homeostase glicêmica, aumentando a glicose hepática e quadros de hiperglicemia (Hanhineva et al., 2010). 
Os polifenóis atuam regulando a glicemia reduzindo a digestão e absorção de glicose no intestino delgado estimula a liberação de insulina pelas células beta-pancreáticas, modulação da liberação de glicose pelo fígado, auxilia na ativação dos receptores de insulina, captação de glicose e vias de sinalização intracelular (Aryaeian et al., 2017). A resistência à insulina pode estar associada ao acúmulo de triglicerídeos no fígado e pode gerar esteatose hepática (Postic \& Girard, 2008).

No estudo de Bose et al. (2008) o chá verde apresenta epigalocatequina e foram capazes de reduzir glicose no sangue e triglicérides no fígado. De acordo com Wolfram et al. (2006), esse efeito da epigalocatequina é devido a redução de ácidos graxos, triglicérides e aumento de insulina. Essa epigalocatequina aumenta a ação de glucoquinase hepática, carnitina palmitoil transferase-1 $\beta$ e reduz a expressão das enzimas gliconeogênicas fosfoenolpiruvato carboxicinase (PEPCK). A melhora da hiperglicemia com epigalocatequina é devido à diminuição da produção endógena de glicose hepática e aumento da liberação de insulina (Wolfram et al., 2006).

Em outro estudo com flavonóides cítricos como hesperidina e naringina também reduzem ácidos graxos livres, triglicérides e colesterol total no plasma e triglicérides hepático. Esses efeitos foram devido ao aumento da glucoquinase hepática e redução da enzima gliconeogênica PEPCK e G6Pase, melhora do metabolismo lipídico (Jung et al., 2006).

A alcachofra é uma planta rica em compostos bioativos e flavonoides, apresentam ação hepatoprotetora, ajudam a remover toxinas e digerem gorduras pela produção da bile do fígado. Contribui para uma função hepática ideal, evitando peroxidação lipídica nas membranas celulares do tecido do fígado e previne dano oxidativo à membrana dos hepatócitos (Salem et al., 2015). Os receptáculos e brácteas são mais ricos em cinarina do que as folhas ou raízes. As alcachofras apresentam compostos importantes considerados hepatoprotetores como ácido (5-O-cafeoilquínico), (ácido clorogênico), ácido 1,3-di-Ocafeoilquínico (cinarina) e ácido 1,5-di-O-cafeoilquínico (El Sayed et al., 2018).

Diferentes polifenóis podem reduzir a saída de glicose hepática, suprimindo a enzima gliconeogênica e aumentando a atividade de glucoquinase para melhorar a glicogênese e a utilização da glicose (Hanhineva et al., 2010).

\subsection{Aumento da eliminação renal}

A insuficiência renal está bem associada às altas taxas de hiperglicemia e pode desencadear complicações macro e microvasculares. Elevados índices glicêmicos geram desequilíbrio redox e inflamação sistêmica e renal sendo um fator preocupante para pacientes com diabetes Mellitus (Amorim et al., 2019).

O comprometimento renal causado pelo diabetes Mellitus é caracterizado por lesão glomerular, aumenta a taxa de filtração glomerular devido a dilatação das arteríolas aferentes. O aumento da hiperglicemia causa vasodilatação das arteríolas, aumenta volume de sangue chegando aos glomérulos, aumento da pressão intraglomerular e aumento taxa de filtração glomerular que consequentemente geram lesões progressivas (Uezima et al., 2012).

Outro fator associado ao aumento da taxa de hiperglicemia em portadores de diabetes Mellitus é a hiperfunção do cotransportador de sódio-glicose (SGLT-2), assim sódio e glicose filtrados são mais reabsorvidos pelos rins e menos sódio-glicose chegam aos túbulos distais gerando vasodilatação das arteríolas aferentes que aumenta a filtração e geram lesão progressiva dos glomérulos (Gallo et al., 2015).

A doença renal por hiperglicemia gera lesões em várias células renais e vasculares (Amorim et al., 2019). Sendo assim, em pacientes diabéticos geralmente encontra proteína na urina indicando lesão renal (Reddy \& Tan, 2020). O controle da glicemia é fundamental para retardar e prevenir a doença renal. Na nefropatia diabética podem ser detectados por dosagem de albumina na urina e creatinina sérica, esses testes devem ser realizados frequentemente. A albumina na urina indica o primeiro estágio da nefropatia com diminuição da taxa de filtração glomerular (Hahr \& Molitch, 2015). 
No estudo de (Sümer et al., 2020), analisaram a capacidade do extrato da alcachofra em ser hepatoprotetor e nefroprotetor. Assim, ratos foram induzidos experimentalmente com paracetamol, e quando foram tratados com as hastes e reptáculo da alcachofra houve melhora dos exames histopatológicos do fígado e rins. Segundo o mesmo autor os marcadores séricos aumentado de BUN e creatinina após induzidos pelo paracetamol, foram melhorados com extrato da alcachofra (Sümer et al., 2020).

Segundo Pires, (2017) os rins apresentam co-transportador de sódio-glicose (SGLT-2) que reabsorve a maior parte da glicose renal. Segundo o mesmo autor, os portadores de diabetes Mellitus (tipo 2) apresentam reabsorção de glicose aumentada nos rins que contribui para elevar índices hiperglicemicos. Assim a inibição desse co-transportador favorece a eliminação de glicose na urina glicosúria auxiliando na redução de glicose no sangue e não sobrecarrega as células beta-pancreáticas (Pires, 2017).

De acordo com Moradi-Marjaneh et al., (2019), os flavonóides florizina tem sido relatado como inibidor de SGLT1 e SGLT2 e seus derivados como canagliflozina, dapagliflozina, empagliflozina, ipragliflozina e ertugliflozina são usados como tratamento de diabetes Mellitus (Moradi-Marjaneh et al., 2019).

Segundo Sato et al., (2007) os extratos de Sophora flavescens são inibidores de SGLT e em sua raiz apresentam quinolizidina alcalóides, flavonóides, benzofurano e triterpenóide. Os compostos maackiaína e formononetina apresentam semelhanças com isoflavonóides e apresentam inibição do co-transportador SGLT2. Assim ficam evidente que os flavonoides apresentam diversas ações na redução da hiperglicemia sendo de grande importância continuar estudos e buscas por mais plantas, compostos naturais que são capazes de inibir o co-transportador (SGLT2).

\section{Conclusão}

De acordo com resultados é necessário considerar a possível ação da alcachofra e seus metabólitos especiais na inibição da alfa-glicosidase, e acrescentar sua importante ação na redução dos radicais livre, redução do glucagon, manutenção hepática do metabolismo glicêmico, proteção das células beta-pancreática, aumento nos níveis de insulina, e captação de glicose nos tecidos, sendo importante fatores na homeostase glicêmica. Por outro lado, a utilização de flavonoides é um dos alvos mais utilizados pela literatura no controle glicêmico a médio e longo prazo. Com a presente revisão é possível concluir que todas as vias mencionadas acima, torna-se algo promissor na busca por tratamento complementar do controle glicêmico de indivíduos portadores do diabetes Mellitus. Sugere como trabalhos futuros, a pesquisa in vivo e in vitro abordando a capacidade da alcachofra em inibir co-transportador renal de sódio e glicose (SGLT2) e sua ação inibindo enzimas digestiva dos carboidratos como alfa-glicosidase, abrindo novas perspectiva na utilização da alcachofra do tratamento complementar do diabetes Mellitu.

\section{Referências}

Abbas, G., Al-Harrasi, A. S., \& Hussain, H. (2017). $\alpha$-Glucosidase Enzyme Inhibitors from Natural Products. In G. Brahmachari (Ed.), Discovery and Development of Antidiabetic Agents from Natural Products: Natural Product Drug Discovery. Elsevier Inc. https://doi.org/10.1016/B978-0-12-8094501.00009-0

Abdel-Salam, O. M. E., Sleem, A. A., \& Shafee, N. (2013). Hepatoprotective effects of Cynara extract and silymarin on carbon tetrachloride-induced hepatic damage in rats. Comparative Clinical Pathology, 23(3). https://doi.org/10.1007/s00580-012-1675-3

Al-Ishaq, R. K., Abotaleb, M., Kubatka, P., Kajo, K., \& Büsselberg, D. (2019). Flavonoids and their anti-diabetic effects: Cellular mechanisms and effects to improve blood sugar levels. Biomolecules, 9(9), 1-35. https://doi.org/10.3390/biom9090430

Amorim, R. G., Guedes, G. da S., Vasconcelos, S. M. de L., \& Santos, J. C. de F. (2019). Doença Renal do Diabetes: Cross-Linking entre Hiperglicemia, Desequilíbrio Redox e Inflamação. Arquivos Brasileiros de Cardiologia, 112(5), 577-587. https://doi.org/10.5935/abc.20190077 
Aryaeian, N., Sedehi, S. K., \& Arablou, T. (2017). Polyphenols and their effects on diabetes management: A review. Medical Journal of the Islamic Republic of Iran, 31(1), 1-14. https://doi.org/10.14196/mjiri.31.134

Ben Salem, M., Ben Abdallah Kolsi, R., Dhouibi, R., Ksouda, K., Charfi, S., Yaich, M., Hammami, S., Sahnoun, Z., Zeghal, K. M., Jamoussi, K., \& Affes, H. (2017). Protective effects of Cynara scolymus leaves extract on metabolic disorders and oxidative stress in alloxan-diabetic rats. BMC Complementary and Alternative Medicine, 17(1), 1-19. https://doi.org/10.1186/s12906-017-1835-8

Bose, M., Lambert, J. D., Ju, J., Reuhl, K. R., Shapses, S. A., \& Yang, C. S. (2008). The major green tea polyphenol, (-)-epigallocatechin-3-gallate, inhibits obesity, metabolic syndrome, and fatty liver disease in high-fat-fed mice. Journal of Nutrition, 138(9), 1677-1683. https://doi.org/10.1093/jn/138.9.1677

Braga, C. de M. (2011). Histórico da utilização de plantas medicinais. 1-24. https://www.bdm.unb.br/bitstream/10483/1856/1/2011_CarladeMoraisBraga.pdf

Campbell, M. K., \& Farrell, S. O. (2016). Bioquímica (2. ed, pp. 1-795). https://integrada.minhabiblioteca.com.br/books/9788522125005.

Carvalheira, J. B. C., Zecchin, H. G., \& Saad, M. J. A. (2002). Vias de Sinalização da Insulina. Arquivos Brasileiros de Endocrinologia \& Metabologia, 46(4), 419-425. https://doi.org/10.1590/s0004-27302002000400013

Cerf, M. E. (2013). Beta cell dysfunction and insulin resistance. Frontiers in Endocrinology, 4(MAR), 1-13. https://doi.org/10.3389/fendo.2013.00037

Christensen, A. A., \& Gannon, M. (2019). The Beta Cell in Type 2 Diabetes. Current Diabetes Reports, 19(9), 1-8. https://doi.org/10.1007/s11892-019-11964

Dragan, S., Andrica, F., Serban, M.-C., \& Timar, R. (2014). Polyphenols-Rich Natural Products for Treatment of Diabetes. Current Medicinal Chemistry, 22(1), 14-22. https://doi.org/10.2174/0929867321666140826115422

El Sayed, A. M., Hussein, R., Motaal, A. A., Fouad, M. A., Aziz, M. A., \& El-Sayed, A. (2018). Artichoke edible parts are hepatoprotective as commercial leaf preparation. Revista Brasileira de Farmacognosia, 28(2), 165-178. https://doi.org/10.1016/j.bjp.2018.01.002

Ernst, H. A., Lo Leggio, L., Willemoës, M., Leonard, G., Blum, P., \& Larsen, S. (2006). Structure of the Sulfolobus solfataricus $\alpha$-Glucosidase: Implications for Domain Conservation and Substrate Recognition in GH31. Journal of Molecular Biology, 358(4), 1106-1124. https://doi.org/10.1016/j.jmb.2006.02.056

Fantini, N., Colombo, G., Giori, A., Riva, A., Morazzoni, P., Bombardelli, E., \& Carai, M. A. M. (2011). Evidence of glycemia-lowering effect by a Cynara scolymus L. extract in normal and obese rats. Phytotherapy Research, 25(3), 463-466. https://doi.org/10.1002/ptr.3285

Fernandes, A. C. F. (2020). Explorando in vitro o efeito antiglicante, inibidor de enzimas digestivas e anti-inflamatório de extratos fenólicos de resíduos agroindustriais. Unicamp, 21(1), 1-9. http://repositorio.unicamp.br/handle/REPOSIP/346970.

Ferrier, D. R. (2018). Bioquímica ilustrada. (7.ed). https://integrada.minhabiblioteca.com.br/books/9788582714867.

Frutos, M. J., Ruiz-Cano, D., Valero-Cases, E., Zamora, S., \& Pérez-Llamas, F. (2018). Artichoke (Cynara scolymus L.). In Nonvitamin and Nonmineral Nutritional Supplements (Nabavi, Se, Issue 2006, pp. 135-138). Elsevier Inc. https://doi.org/10.1016/B978-0-12-812491-8.00018-7

Galicia-Garcia, U., Benito-Vicente, A., Jebari, S., Larrea-Sebal, A., Siddiqi, H., Uribe, K. B., Ostolaza, H., \& Martín, C. (2020). Pathophysiology of type 2 diabetes mellitus. International Journal of Molecular Sciences, 21(17), 1-34. https://doi.org/10.3390/ijms21176275

Gallo, L. A., Wright, E. M., \& Vallon, V. (2015). Probing SGLT2 as a therapeutic target for diabetes: Basic physiology and consequences. Diabetes and Vascular Disease Research, 12(2), 78-89. https://doi.org/10.1177/1479164114561992

Ghani, U. (2020a). Introduction, rationale and the current clinical status of oral $\alpha$-glucosidase inhibitors. In U. Ghani (Ed.), Alpha-Glucosidase Inhibitors (pp. 1-15). Elsevier. https://doi.org/10.1016/b978-0-08-102779-0.00001-0

Ghani, U. (2020b). Polyphenols. In U. Ghani (Ed.), Alpha-Glucosidase Inhibitors (pp. 61-100). Elsevier. https://doi.org/10.1016/B978-0-08-102779-0.000034

Grandi, T. S. M. (2014). Tratado das plantas Medicinais: Mineiras, Nativas e Cultivadas (pp. 7-1186).

Hahr, A. J., \& Molitch, M. E. (2015). Management of diabetes mellitus in patients with chronic kidney disease. Clinical Diabetes and Endocrinology, 1(2), 19. https://doi.org/10.1186/s40842-015-0001-9

Hanhineva, K., Törrönen, R., Bondia-Pons, I., Pekkinen, J., Kolehmainen, M., Mykkänen, H., \& Poutanen, K. (2010). Impact of dietary polyphenols on carbohydrate metabolism. International Journal of Molecular Sciences, 11(4), 1365-1402. https://doi.org/10.3390/ijms11041365

Hosseini, S. E., Mousaei, S., \& Tavakoli, F. (2015). Effect of hydro alcoholic extract of artichoke on diabetes treatment and liver enzymes in diabetic adult male rats. Advanced Herbal Medicine, 1(1), 17-21. http://herbmed.skums.ac.ir/article_11234_1514.html

Idf, I. D. F. (2019). International Diabetes Federation. In The Lancet $\left(9^{\circ}\right)$. https://www.idf.org/e-library/welcome/copyright-permission.html

Jung, U. J., Lee, M. K., Park, Y. B., Kang, M. A., \& Choi, M. S. (2006). Effect of citrus flavonoids on lipid metabolism and glucose-regulating enzyme mRNA levels in type-2 diabetic mice. International Journal of Biochemistry and Cell Biology, 38(7), 1134-1145. https://doi.org/10.1016/j.biocel.2005.12.002

Klover, P. J., \& Mooney, R. A. (2004). Hepatocytes: Critical for glucose homeostasis. International Journal of Biochemistry and Cell Biology, 36(5), 753758. https://doi.org/10.1016/j.biocel.2003.10.002

Lattanzio, V., Kroon, P. A., Linsalata, V., \& Cardinali, A. (2009). Globe artichoke: A functional food and source of nutraceutical ingredients. Journal of Functional Foods, 1(2), 131-144. https://doi.org/10.1016/j.jff.2009.01.002 
Lyra, R., Oliveira, M., Lins, D., Cavalcanti, N., Gross, J. L., Maia, F. F. R., Araújo, L. R., Yafi, M., Guimarães, F. P. D. M., Takayanagui, A. M. M., Lucena, J. B. D. S., Golbert, A., Campos, M. A. a., Saúde, M. da, Sartorelli, D. S., Franco, L. J., Prevenção, E., \& Marcondes, J. a. M. (2020). Sociedade Brasileira de Diabetes. In E. C. Clannad (Ed.), Diabetes Mellitus Tipo 1 e Tipo2 (Vol. 5, Issue 3).

Mahboubi, M. (2018). Cynara scolymus (artichoke) and its efficacy in management of obesity. Bulletin of Faculty of Pharmacy, Cairo University, 56(2), 115120. https://doi.org/10.1016/j.bfopcu.2018.10.003

Malta, D. C., \& Silva Jr, J. B. da. (2013). O Plano de Ações Estratégicas para o Enfrentamento das Doenças Crônicas Não Transmissíveis no Brasil e a definição das metas globais para o enfrentamento dessas doenças até 2025: uma revisão. Epidemiologia e Serviços de Saúde, 22(1), 151-164. https://doi.org/10.5123/s1679-49742013000100016

Mejri, F., Baati, T., Martins, A., Selmi, S., Luisa Serralheiro, M., Falé, P. L., Rauter, A., Casabianca, H., \& Hosni, K. (2020). Phytochemical analysis and in vitro and in vivo evaluation of biological activities of artichoke (Cynara scolymus L.) floral stems: Towards the valorization of food by-products. Food Chemistry, 333(July), 127-506. https://doi.org/10.1016/j.foodchem.2020.127506

Mishra, S., Singh, A. S., Mishra, N., Pandey, H., \& Tiwari, V. K. (2017). Carbohydrate-Based Antidiabetic Agents from Nature. In Discovery and Development of Antidiabetic Agents from Natural Products: Natural Product Drug Discovery. Elsevier Inc. https:/doi.org/10.1016/B978-0-12-809450$1.00005-3$

Moore, M. C., Coate, K. C., Winnick, J. J., An, Z., \& Cherrington, A. D. (2012). Regulation of Hepatic Glucose Uptake and Storage In Vivo1,2. American Society for Nutrition, 286-294. https://doi.org/10.3945/an.112.002089

Moradi-Marjaneh, R., Paseban, M., \& Sahebkar, A. (2019). Natural products with SGLT2 inhibitory activity: Possibilities of application for the treatment of diabetes. Phytotherapy Research, 33(10), 2518-2530. https://doi.org/10.1002/ptr.6421

Muccilli, V., Cardullo, N., Spatafora, C., Cunsolo, V., \& Tringali, C. (2017). $\alpha$-Glucosidase inhibition and antioxidant activity of an oenological commercial tannin. Extraction, fractionation and analysis by HPLC/ESI-MS/MS and 1H NMR. Food Chemistry, 215, 50-60. https://doi.org/10.1016/j.foodchem.2016.07.136

Mueckler, M., \& Thorens, B. (2013). The SLC2 (GLUT) family of membrane transporters. Molecular Aspects of Medicine, 34(2-3), 121-138. https://doi.org/10.1016/j.mam.2012.07.001

Pereira, A., Shitsuka, D., Parreira, F., \& Shitsuka, R. (2018). Metodologia da pesquisa Científica. In Metodologia da Pesquisa Científica (1. ed). https://repositorio.ufsm.br/bitstream/handle/1/15824/Lic_Computacao_Metodologia-Pesquisa-Cientifica.pdf?sequence=1. Acesso em: 28 março 2020.

Pires, L. da S. (2017). Efeito da inibição do SGLT-2 pela dapagliflozina no cérebro e na funcionalidade hipotalâmica em humanos e camundongos. Unicamp, 1-14. http://repositorio.unicamp.br/jspui/handle/REPOSIP/330857

Postic, C., \& Girard, J. (2008). Contribution of de novo fatty acid synthesis to hepatic steatosis and insulin resistance: Lessons from genetically engineered mice. Journal of Clinical Investigation, 118(3), 829-838. https://doi.org/10.1172/JCI34275

Rastija, V., Bešlo, D., \& Nikolić, S. (2012). Two-dimensional quantitative structure-activity relationship study on polyphenols as inhibitors of a-glucosidase. Medicinal Chemistry Research, 21(12), 3984-3993. https://doi.org/10.1007/s00044-011-9938-0

Reddy, S. S. K., \& Tan, M. (2020). Diabetes mellitus and its many complications. In M. Tan (Ed.), Diabetes Mellitus (pp. 1-18). Elsevier Inc. https://doi.org/10.1016/b978-0-12-820605-8.00001-2

Rezazadeh, K., \& Ebrahimi-Mameghani, M. (2020). Artichoke leaf extract and use in metabolic syndrome as an antioxidant. In Pathology Oxidative Stress and Dietary Antioxidants (Pathology, pp. 169-177). Preedy, Victor R. https://doi.org/10.1016/b978-0-12-815972-9.00016-0

Rosak, C., \& Mertes, G. (2012). Critical evaluation of the role of acarbose in the treatment of diabetes: Patient considerations. Diabetes, Metabolic Syndrome and Obesity: Targets and Therapy, 5, 357-367. https://doi.org/10.2147/dmso.s28340

Salem, M. Ben, Affes, H., Ksouda, K., Dhouibi, R., Sahnoun, Z., Hammami, S., \& Zeghal, K. M. (2015). Pharmacological Studies of Artichoke Leaf Extract and Their Health Benefits. Plant Foods for Human Nutrition, 70(4), 441-453. https://doi.org/10.1007/s11130-015-0503-8

Salles, B. C. C., Silva, M. A. da, Taniguthi, L., Ferreira, J. N., Quintino da Rocha, C., Vilegas, W., Dias, P. H., Pennacchi, P. C., Maris da Silveira Duarte, S., Rodrigues, M. R., Lima Brigagão, M. R. P., \& Borges de Araújo Paula, F. (2020). Passiflora edulis Leaf Extract: Evidence of antidiabetic and antiplatelet effects in rats. Biological and Pharmaceutical Bulletin, 43(1), 169-174. https://doi.org/10.1248/bpb.b18-00952

Salles, B. C. C., Terra, M. C., \& Paula, F. B. de A. (2019). Sinalização mediada pela insulina em vias anabólicas. Generalist Pharmacy Journal, 1(2), 25-45.

Santos, C. M. M., Freitas, M., \& Fernandes, E. (2018). A comprehensive review on xanthone derivatives as $\alpha$-glucosidase inhibitors. European Journal of Medicinal Chemistry, 157, 1460-1479. https://doi.org/10.1016/j.ejmech.2018.07.073

Sato, S., Takeo, J., Aoyama, C., \& Kawahara, H. (2007). Na+-Glucose cotransporter (SGLT) inhibitory flavonoids from the roots of Sophora flavescens. Bioorganic and Medicinal Chemistry, 15(10), 3445-3449. https://doi.org/10.1016/j.bmc.2007.03.011

Saucier, C. (2013). Caracterização química das folhas de alcachofra (Cynara scolymus L.) por cromatografia gasosa monodimensional e Bidimensional abrangente. Instituto de Química-Universidade Federal Do Rio Grande Do Sul, 1-109. http://hdl.handle.net/10183/80428

Sheliya, M. A., Rayhana, B., Ali, A., Pillai, K. K., Aeri, V., Sharma, M., \& Mir, S. R. (2015). Inhibition of $\alpha$-glucosidase by new prenylated flavonoids from euphorbia hirta L. herb. Journal of Ethnopharmacology, 176, 1-8. https://doi.org/10.1016/j.jep.2015.10.018

Sümer, E., Senturk, G. E., Demirel, Ö. U., \& Yesilada, E. (2020). Comparative biochemical and histopathological evaluations proved that receptacle is the most effective part of Cynara scolymus against liver and kidney damages. Journal of Ethnopharmacology, 249(November), 112-458. https://doi.org/10.1016/j.jep.2019.112458 
Research, Society and Development, v. 10, n. 7, e6610716273, 2021

(CC BY 4.0) | ISSN 2525-3409 | DOI: http://dx.doi.org/10.33448/rsd-v10i7.16273

Taslimi, P., \& Gulçin, İ. (2017). Antidiabetic potential: in vitro inhibition effects of some natural phenolic compounds on $\alpha$-glycosidase and $\alpha$-amylase enzymes. Journal of Biochemical and Molecular Toxicology, 31(10), 1-6. https://doi.org/10.1002/jbt.21956

Uezima, C. B. B., Zanella, M. T., Sachs, A., Pimazzoni Netto, A., \& Zach, P. L. (2012). Efeitos do controle glicêmico obtido em curto prazo sobre a microalbuminúria e a filtração glomerular em pacientes diabéticos do tipo 2 com controle glicêmico precário. Jornal Brasileiro de Nefrologia, 34(2), 130-138. https://doi.org/10.1590/s0101-28002012000200005

Viegas Jr, C., Bolzani, V. da S., \& Barreiro, E. J. (2006). Os produtos naturais e a química medicinal moderna. Química Nova, 29 (2), 326-337. https://doi.org/10.1590/s0100-40422006000200025

Wolfram, S., Raederstorff, D., Preller, M., Wang, Y., Teixeira, S. R., Riegger, C., \& Weber, P. (2006). Epigallocatechin gallate supplementation alleviates diabetes in rodents. Journal of Nutrition, 136(10), 2512-2518. https://doi.org/10.1093/jn/136.10.2512

World Health Organization. (2014). Global Status Report On Noncommunicable Diseases 2014. 7-302.

Zuorro, A., Maffei, G., \& Lavecchia, R. (2016). Reuse potential of artichoke (Cynara scolimus L.) waste for the recovery of phenolic compounds and bioenergy. Journal of Cleaner Production, 111, 1-6. https://doi.org/10.1016/j.jclepro.2015.06.011 